\title{
EL NUEVO PUENTE SOBRE EL RÍO PISUERGA DE LA RONDA SUR DE VALLADOLID
}

\author{
(THE NEW BRIDGE OVER PISUERGA RIVER IN THE SOUTH \\ ROAD STRETCH OF VALLADOLID)
}

Juan J. Arenas de Pablo, Ingeniero de Caminos, Canales y Puertos

Univ. Politécnica de Cantabria. Pte. de Apia XXI, S.A.

ESPAÑA

Fecha de recepción: $18-$ II-98

$562-153$

\begin{abstract}
RESUMEN
Se describe el proyecto de obra del nuevo puente sobre el río Pisuerga.
\end{abstract}

Teniendo en cuenta lo peculiar de la topografia del lugar, se sugiere la idea de puente atirantado, planteándose su construcción en el tipo de apoyos que ha de presentar dicho puente y su sistema constructivo, considerando, de manera especial, su durabilidad.

\section{SUMMARY}

The project for the work of a new bridge over Pisuerga river is described in this article.

Considering how peculiar the topography of the place is, the idea of a truss-bridge is suggested, planning its construction thinking of the bearings that have to support the bridge and its constructive system, intending first its durability.

\section{Idea general}

La Ronda Sur cruza el río Pisuerga aguas abajo de Valladolid en un punto del cauce de unos 100 metros de agua y de marcado carácter asimétrico: mientras en la margen izquierda la orilla desciende suavemente hacia el agua, existe en la derecha un borde bastante cortado, en cuya inmediación discurre, paralela al río, la antigua carretera de Salamanca, hoy convertida en avenida.

A la hora de proyectar esta obra por encargo del Ayuntamiento de Valladolid, se ha tenido muy presente el carácter urbano del puente, así como el papel de vía principal que esta nueva Ronda iba a desempeñar. En consecuencia, y de acuerdo con el Ayuntamiento, se planteó sobre el Pisuerga un puente atirantado de configuración bastante especial.

El carácter urbano de la Ronda y del puente llevaron al Ayuntamiento a plantear, además de los 4 carriles de tráfico separados por mediana, aceras y bandas para ciclistas, condiciones a las que el puente trató de adaptarse.

La obra, incluida dentro de la Fase II de la Ronda Sur, ha sido financiada por la Consejería de Fomento de la Junta de Castilla y León, habiendo sido adjudicada a la UTE formada por las empresas Ferrovial y Zarzuela en el año 1996, cuyo plazo de ejecución termina en marzo de 1999. Al tiempo, la Junta honraba al autor del artículo con el encargo de la Dirección de obra, lo que, aparte de cargarle con una responsabilidad absoluta, puso en sus manos la totalidad de decisiones de la misma que, como se sabe, condicionan la calidad final de la obra construida tanto como el mismo proyecto.

\section{Descripción del puente}

De esa topografía asimétrica, que invitaba a establecer un apoyo importante en la margen izquierda, espaciosa y reposada, pero que, por contra, instaba a apoyar con la mayor ligereza en la derecha, nació una propuesta de puente atirantado, asimétrico, con mástiles en sólo esa margen izquierda. Buscando cruzar el río de un único salto, se planteó el apoyo de ese mástil fuera del agua. Apareció entonces como necesaria una luz de unos 120 metros, luz que conlleva la exigencia de un vano de contrarresto de, al menos, 30 metros, que se acomodaba perfectamente en la orilla izquierda, pero para el que no había espacio en la derecha.

La idea de puente atirantado surge de modo natural en esta topografía concreta: por un lado, se deseaba saltar el agua 
de un golpe; por otro, ni la configuración del valle ni el terreno de cimentación invitaban a establecer un arco, ni se consideraba un buen encaje a la solución bowstring de arco atirantado por el tablero. El atirantamiento aparece en esta forma de valle como una solución natural a esa postura básica de salvar el cauce sin apoyos intermedios.

En paralelo con esa concepción longitudinal, se plantea la organización transversal de la estructura y de sus sistemas de atirantamiento. Y se decide proponer un tablero que en sección tipo, cubriendo un ancho total de 31 metros, aloje las dos calzadas dobles, de $850 \mathrm{~cm}$ cada una; una mediana central de 2 metros de ancho y aceras de 6 metros, donde se incluyen las pistas de ciclistas.

Para ello se dispusieron sendos planos verticales de atirantamiento alojados en el interior de cada acera y situados de modo que los tirantes hagan el papel de plano transparente que separa la acera de peatones, externa al puente, de la pista de ciclistas, contigua a la calzada.

\subsection{Sistema mástil-vela}

La idea inicial de proyecto está basada en lo que hemos llamado sistema mástil-vela. Cada mástil es una pieza situada en ese plano vertical, que va a nacer de la misma acera de forma bastante peculiar y que va a componer una pieza estructural y arquitectónica de primera magnitud. Por ello, y desde el primer momento, se imaginó inclinado en el plano longitudinal, creciendo en desplome hacia el río como si deseara facilitar el amarre de los tirantes que desde su cabeza van a suspender al tablero. Con incidencia de su mayor dificultad constructiva, el mástil inclinado, con talud 3/1, quedará amarrado hacia atrás por un conjunto de tirantes de contrarresto y su trabajo estructural será básicamente el de una pieza comprimida. Y la pareja de mástiles a uno y otro lado del puente, paralelos e inclinados hacia adelante, ofrecerán una imagen que tiene algo que ver con un par de robustas lanzas apoyadas en el suelo.

La fuerte asimetría de la organización elegida lleva a la necesidad de resistir una importante reacción vertical ascendente en el extremo dorsal de la obra, lo que puede conseguirse bien con el empleo de barras de anclaje al terreno profundo, bien recurriendo a un macizo de contrapeso. A la vista de la incertidumbre de corrosión y durabilidad que siempre suponen los anclajes en tierra, se eligió decididamente el último. Y se hizo un esfuerzo en extraer a ese contrapeso todo el valor arquitectónico y expresivo posibles, de modo que, lejos de buscar cualquier ocultación como macizo indeseable, se convierta en uno de los puntos importantes de la composición arquitectónica de esta obra.

Sin embargo, esta idea inicial que era la de plantear tirantes de contrarresto entre cabeza de cada mástil y contrapeso dorsal fue pronto sustituida por la menos conocida de establecer una pieza de hormigón que, lanzándose hacia arriba como vector inclinado, atara esos dos puntos. Idea que germinó al comprobar que, en situaciones de máxima sobrecarga en el vano principal y como consecuencia de la inevitable elongabilidad de esos tirantes de amarre, aparecían importantes flexiones positivas en los nervios del tablero. La sustitución de esos tirantes de acero por las piezas de hormigón pretensado supuso, gracias al aumento de rigidez axil de estas últimas, reducir tanto la flexión como la flecha vertical que esa sobrecarga producía a la quinta parte.

Había nacido así las que se acostumbró a llamar "velas", piezas de canto variable, cuyas proyecciones horizontal y vertical alcanzan medidas de 48 y 34 metros, con una longitud inclinada de unos 58 metros, cuyo eje tiene una pendiente del $70 \%$. Y, en paralelo con ellas, nacía también el concepto de cabeza de anclaje de los tirantes, que hubo que pasar como pieza de coronación del mástil y de fusión de éste con la vela. Las cabezas, cuya forma tiene bastante de cuerpo vivo, responden a la triple función de alojar en su canal posterior los anclajes activos de los tirantes, de recibir los anclajes de los tendones de pretensado de la vela y de transmitir al mástil la fuerza de compresión resultante del tiro de tirantes y de la tracción compensadora de la vela.

En suma, se terminó disponiendo de un caballete de hormigón, suma del mástil comprimido y de la vela traccionada, que es capaz de resistir por esfuerzo axil el tiro variable de los tirantes, y ello con muy pequeña deformación axil de ambas piezas. Son piezas que forman el mecanismo resistente primario del puente $\mathrm{y}$, por tanto, resultan esenciales para la seguridad estructural. Pero, además, su trascendencia arquitectónica no escapa a nadie. Razón por la que se trató de plantearlos como piezas de valor escultórico: canto linealmente variable, con sección que dispone de importantes rehundidos y con contactos muy cuidados entre unos y otros, con el objetivo de buscar para la cabeza una forma en alzado y sección que englobe y acoja al mástil y a la vela.

Las dimensiones de estas piezas son en todo inusuales y su dificultad de ejecución considerable. Por ejemplo:

- El mástil dispone de una pendiente en el plano longitudinal de $3 / 1$. La vela, como se ha dicho, de 7/10.

- Con un ancho constante de $240 \mathrm{~cm}$, los cantos de la vela oscilan entre 284 y $535 \mathrm{~cm}$.

- Los del mástil inclinado entre 4 y 5 metros y medio. Su ancho, constante, es de 2,80 metros.

- La cabeza se organiza como una pieza sandwich, con dos "tablas" externas de $110 \mathrm{~cm}$ de grueso y un panel interno rehundido de $80 \mathrm{~cm}$, siendo su ancho total de 3 metros. 

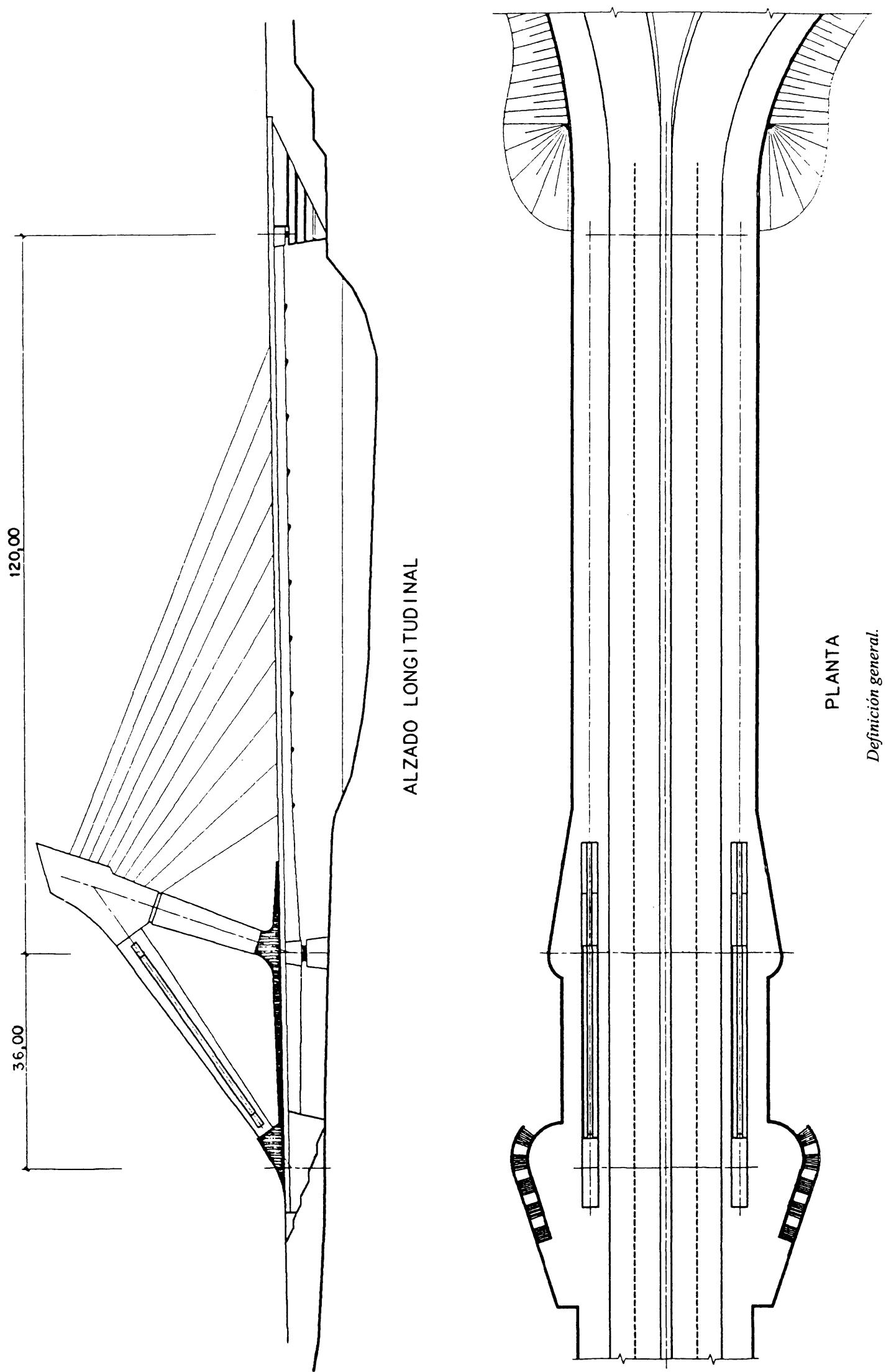


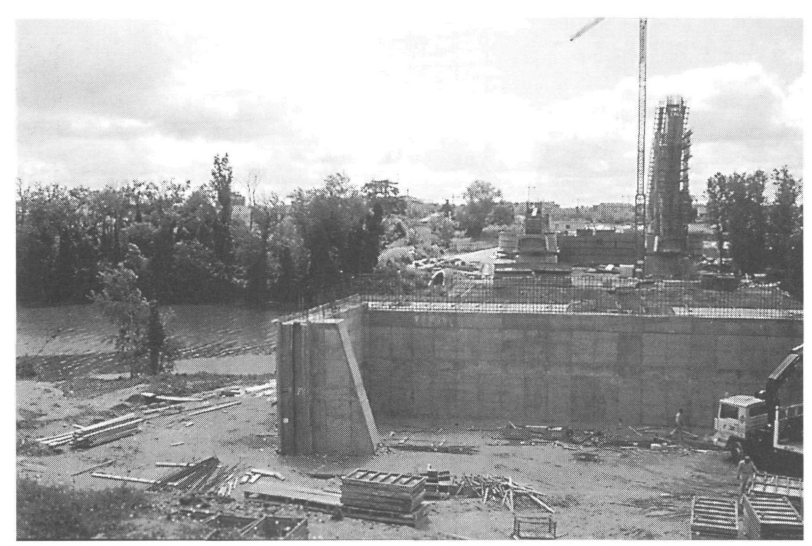

Estribo margen derecha.

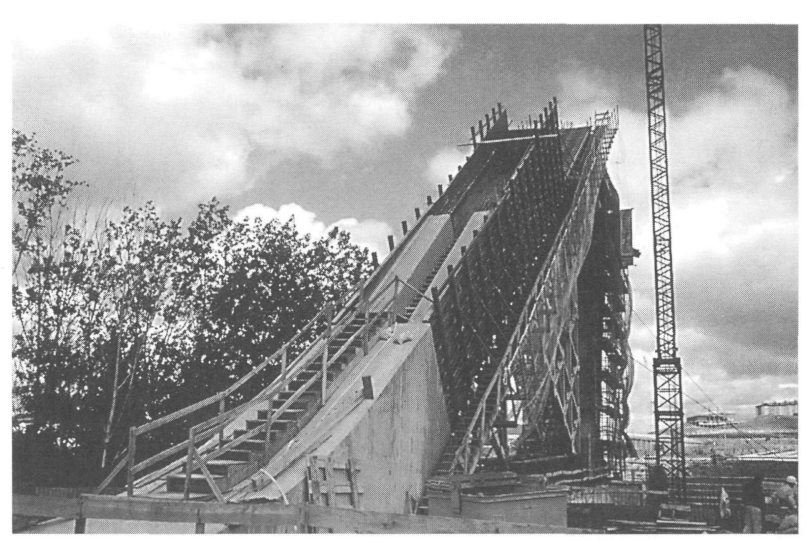

Vela en construcción.

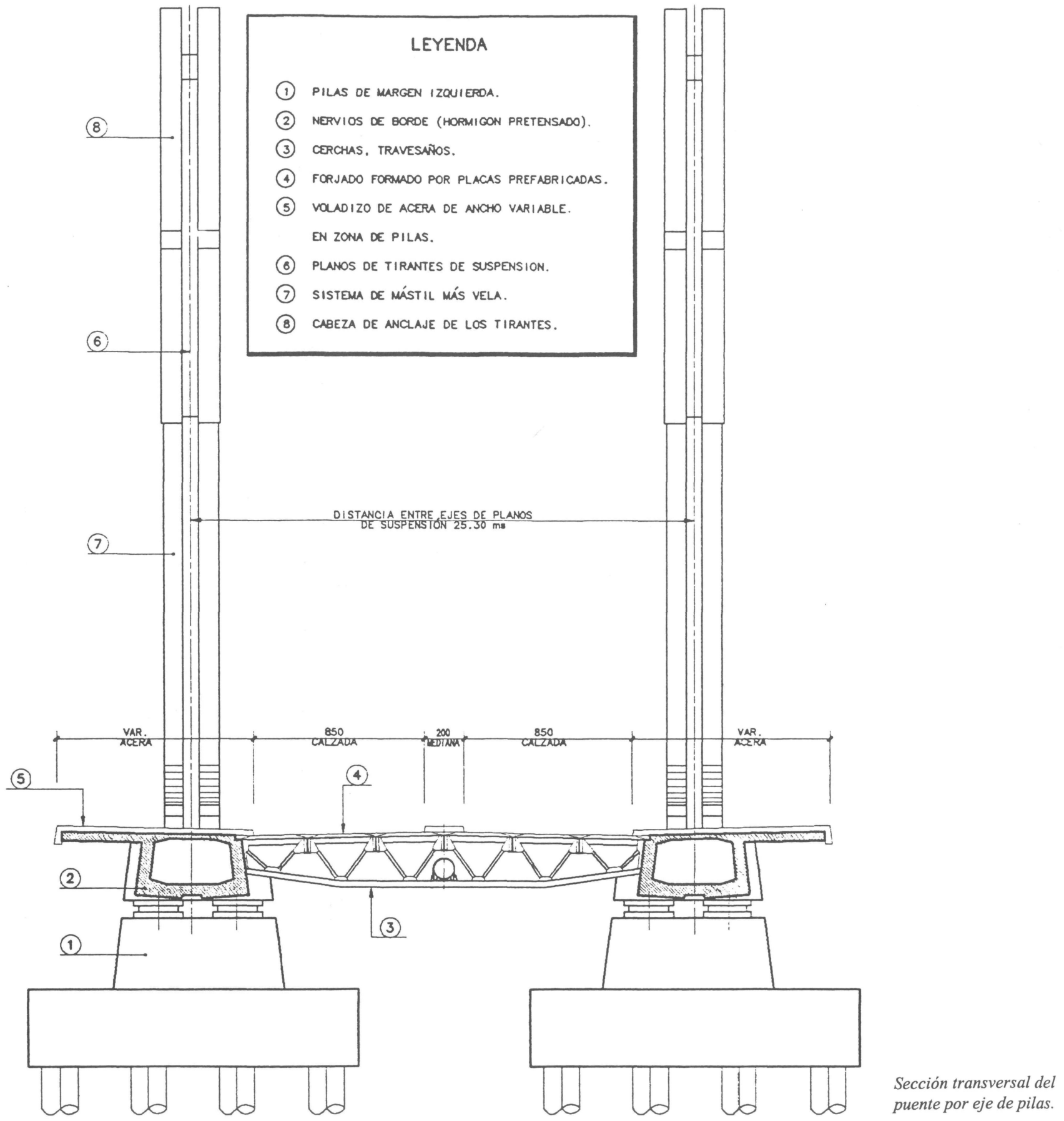


- La máxima dimensión vertical de la cabeza es del orden de los 20,5 metros.

- El volumen de hormigón de la misma cabeza alcanza los $443 \mathrm{~m}^{3}$.

- El vértice más alto de esa cabeza queda a una altura de unos 42 metros sobre el tablero.

La organización final del puente en sentido longitudinal es la de una estructura continua de dos vanos, con luces de 120 metros el principal y 36 metros el lateral de contrarresto. En alzado, existen entonces 3 únicos apoyos que son:

1. El estribo margen izquierda, llamado contrapeso, y que consiste en un gran bloque de hormigón cimentado en pilotes profundos, al que queda empotrado el tablero.

2. La pila central (o mejor, las dos pilas), también en la orilla izquierda, que dan apoyo directo a cada nervio de borde en la vertical de acometida del eje del mástil, recibiendo así toda su carga axil a través de un par de placas de apoyo.

3. El estribo de margen derecha, compuesto por muro frontal y muros en vuelta, de formas cuidadas.

\section{2. Organización del tablero}

Con dos planos de suspensión espaciados transversalmente 24,30 metros, el tablero se organiza a base de sendos nervios longitudinales en ambos bordes, vigas transversales, en forma de cerchas que, trabajando con esa luz de 25 metros, van a transmitir a los nervios las cargas del tablero y un piso, apoyo directo de los vehículos y peatones que, a su vez, transmitirá las cargas del tráfico a esas cerchas transversales. Sistema relativamente complejo que se describe a continuación:

- Los nervios de borde de hormigón, directamente suspendidos de los tirantes inclinados, constituyen las almas resistentes del tablero y, además, componen la misma fachada del puente. De ahí su sección hueca, de aspecto trapecial, con dos almas y formas muy cuidadas, incluyendo un rehundido longitudinal de su tabla inferior que nace del deseo de alojar con la mayor limpieza los anclajes inferiores de tirantes. Con dimensiones exteriores de $560 \mathrm{~cm}$ de ancho por 3 metros de canto, los nervios disponen de suficiente rigidez de flexión y torsión para su trabajo estructural.

- Nervios que ofrecen sección constante en casi todo el desarrollo del vano principal, planteándose un canto linealmente variable en los 24 metros inmediatos a la sección de apoyo en pila, donde se alcanza un canto de 3,45 metros, que con una ligera variación se mantiene a lo largo del vano lateral.
- La suspensión consiste en 10 tirantes en abanico que, arrancando de la cabeza del mástil, van a anclarse a puntos de cada nervio de borde espaciados 9 metros en sentido longitudinal. Distancia ésta que fue elegida como óptima desde el punto de vista del número de tirantes y de su efecto en el alzado del puente, pero que ha conllevado alguna dificultad a la hora de organizar la estructura del tablero.

- Porque, buscando una transmisión lo más directa de la carga vertical del piso del puente a los tirantes, las cerchas transversales se sitúan en los nudos teóricos de anclaje de tirante, dando lugar a luces locales de 9 metros para ese tablero. Cerchas que han de salvar una luz importante $(25,30$ metros) y que se han planteado de acero, con alzado en forma de vientre de pez y canto máximo en el eje del puente, con una cabeza superior que dispone de conectadores y funciona, junto a la losa de hormigón de $18 \mathrm{~cm}$ de grueso, como pieza mixta.

- Cerchas que se empotran a flexión en ambos nervios de borde, donde ese grado de empotramiento va a depender de la rigidez torsional de los últimos, lo que al fin tiene que ver con la mayor o menor proximidad de la cercha considerada a las secciones extremas de pila o de apoyo en estribo, donde esa rigidez del nervio de borde al giro torsional es total. Es esa integral de cargas torsoras la que conduce a los momentos torsores que ha de soportar cada nervio de borde, que sólo podrá descargar en las secciones donde dispone de empotramiento torsional: las de apoyo en contrapeso, pila y estribo de margen derecha.

- La triangulación de la cercha conduce a nudos del par superior espaciados $342 \mathrm{~cm}$ entre sí, puntos a los que hacemos acometer un conjunto de 5 largueros, vigas longitudinales de chapa de acero de unos $70 \mathrm{~cm}$ de canto que, dotadas de conectadores, funcionan como piezas mixtas continuas con la losa superior de hormigón.

- Tratando de eliminar la necesidad de encofrado para la ejecución de la losa del tablero, se planteó componer su piso con placas de hormigón prefabricadas, de sólo $18 \mathrm{~cm}$ de canto. Ello exige luces menores de 9 metros y de ahí que entre cada dos cerchas se establezca una pieza transversal de acero, denominada "travesaño", en forma de viga doble $\mathrm{T}$ de chapa, también mixta, que funciona apoyada en el conjunto de largueros que enlazan las sucesivas cerchas.

- Al final, se dispone de una retícula de módulos de $342 \times 450 \mathrm{~cm}$, dispuesta para recibir placas prefabricadas que se apoyan en los bordes de las platabandas superiores de la cercha, los largueros y el travesaño, cuyos bordes se conforman para hacer posible el llenado de franjas de hormigón in situ que, con la armadura vista de esas placas, permite alcanzar una continuidad y monolitismo totales.

-La planta espejo del puente ofrece así una imagen artesonada de máxima limpieza, con una retícula formada 
ธี
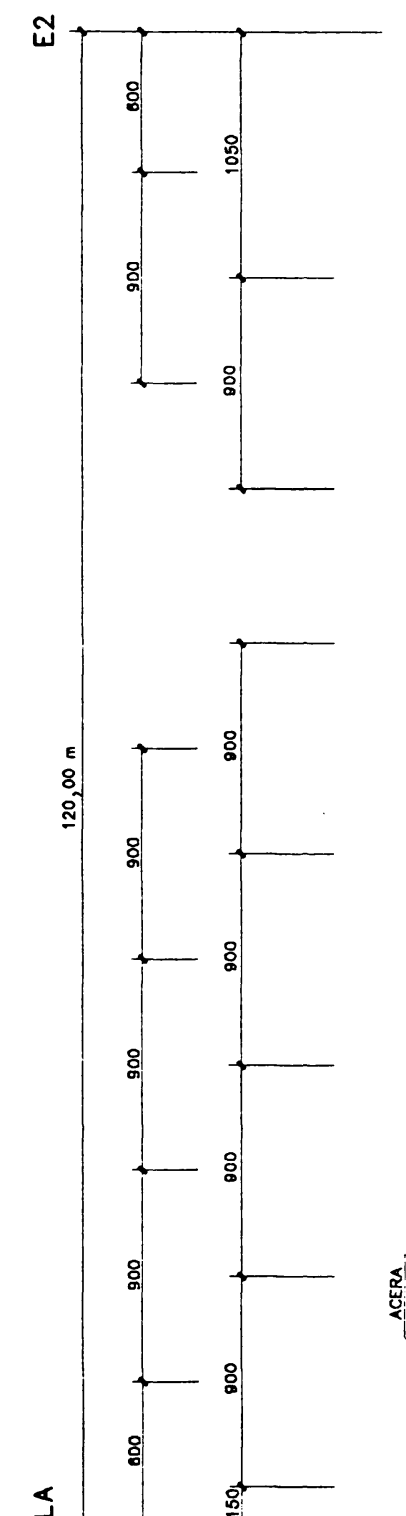

高

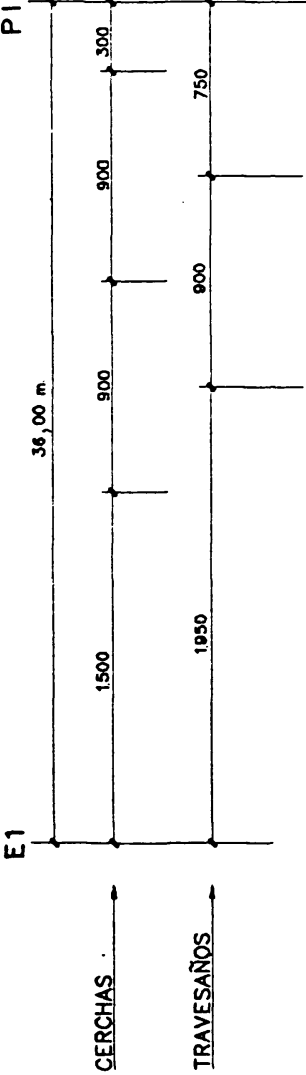

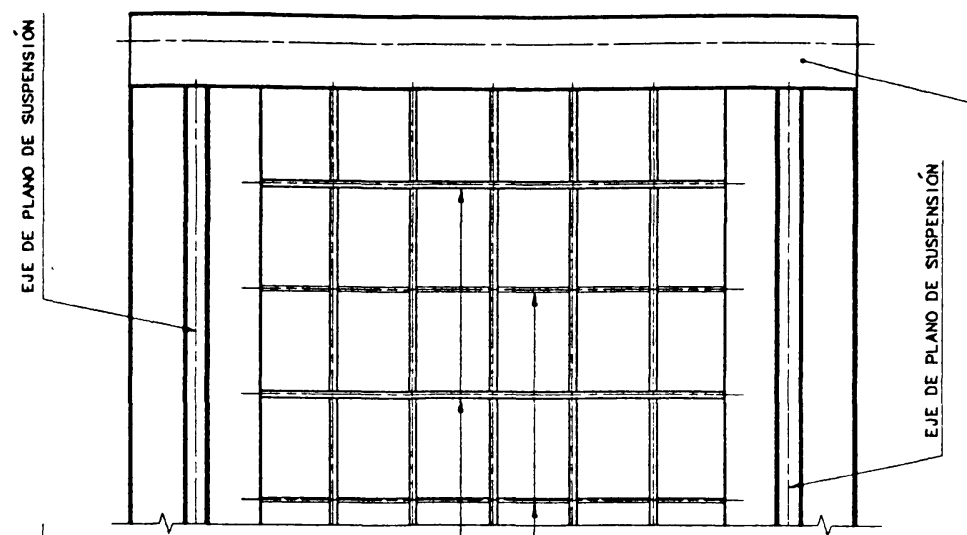
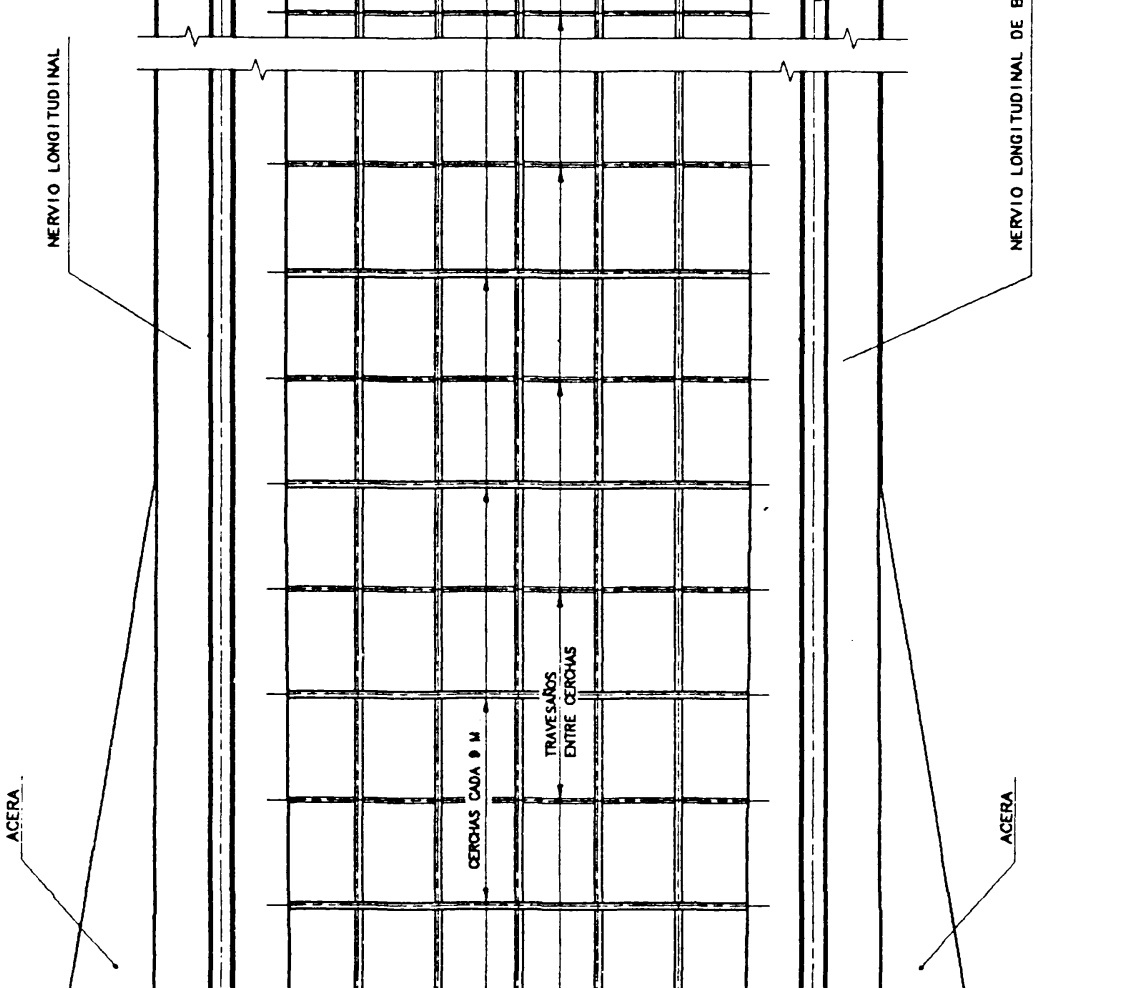

0
8
5
$\frac{8}{2}$
$\frac{5}{2}$ 


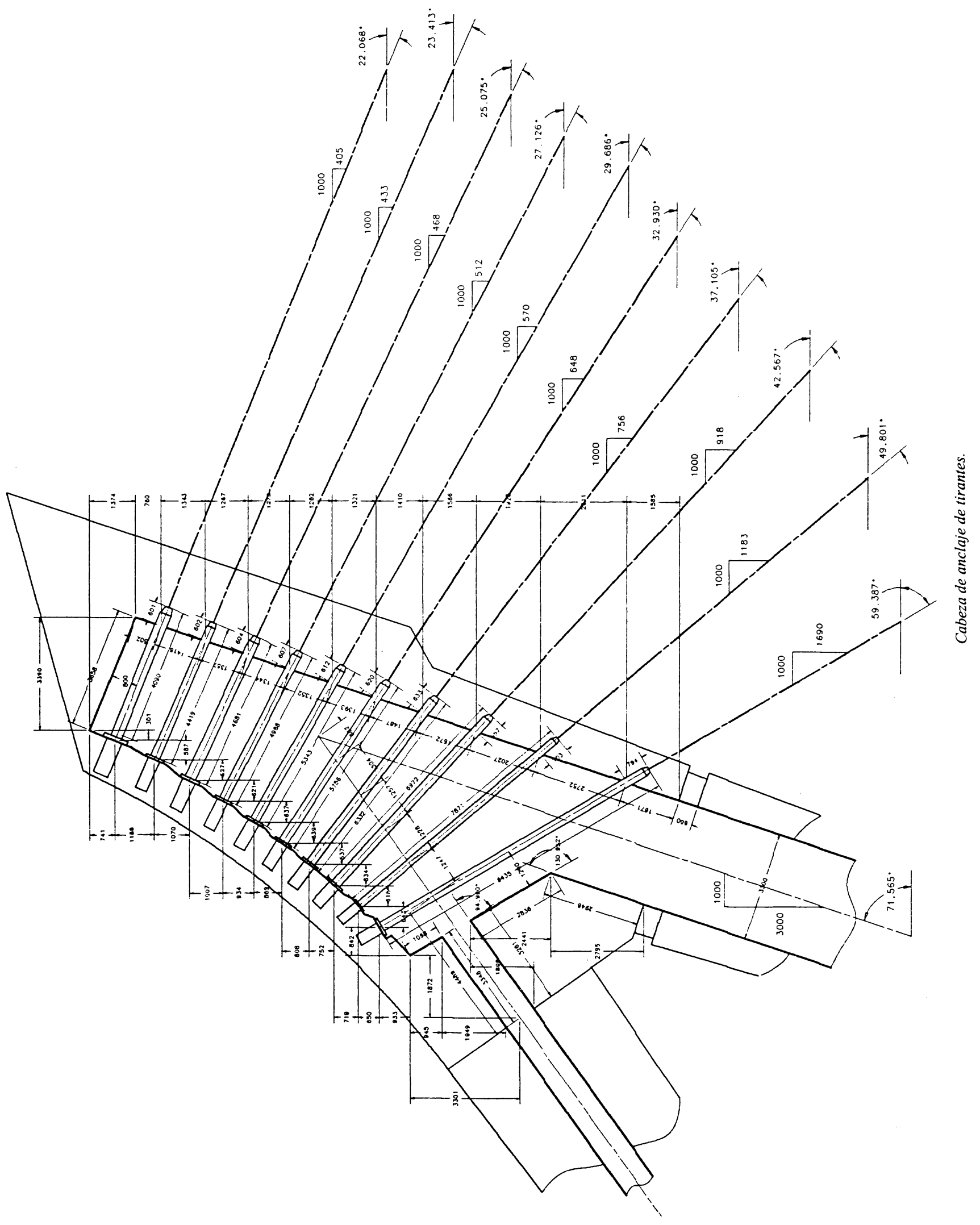




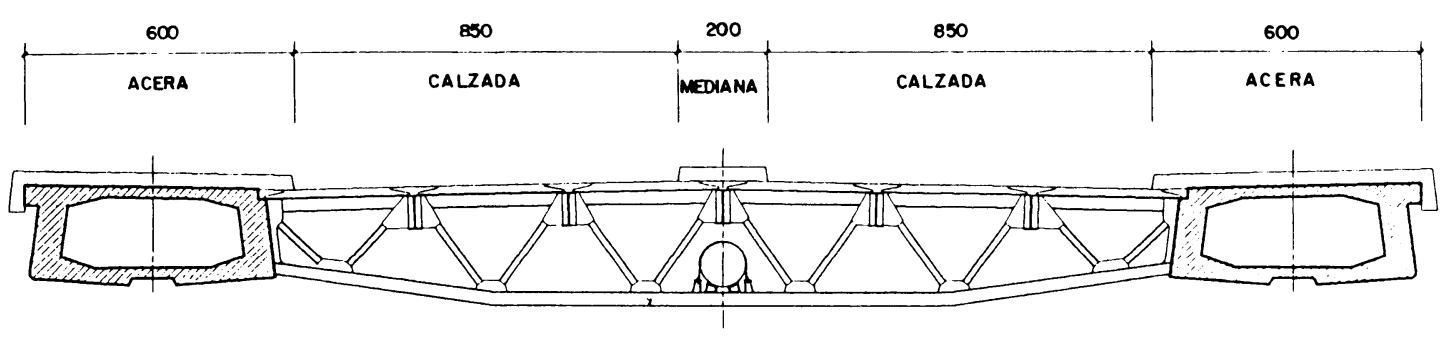

SECCIÓN TRANSVERSAL DEL PUENTE POR VANO PRINCIPAL

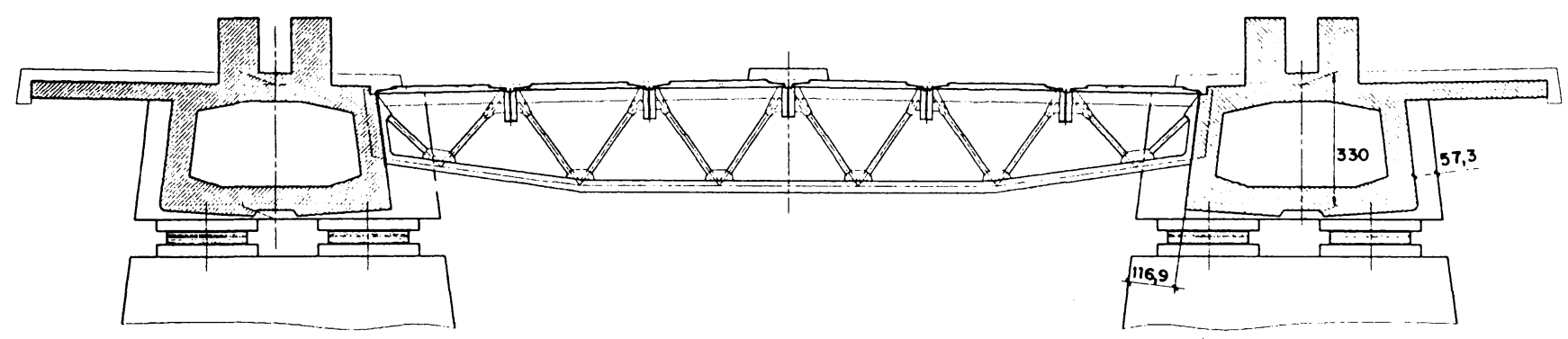

SEÇCIÓN TRANSVERSAL DEL PUENTE PROXIMA A PILA

Secciones transversales del puente.

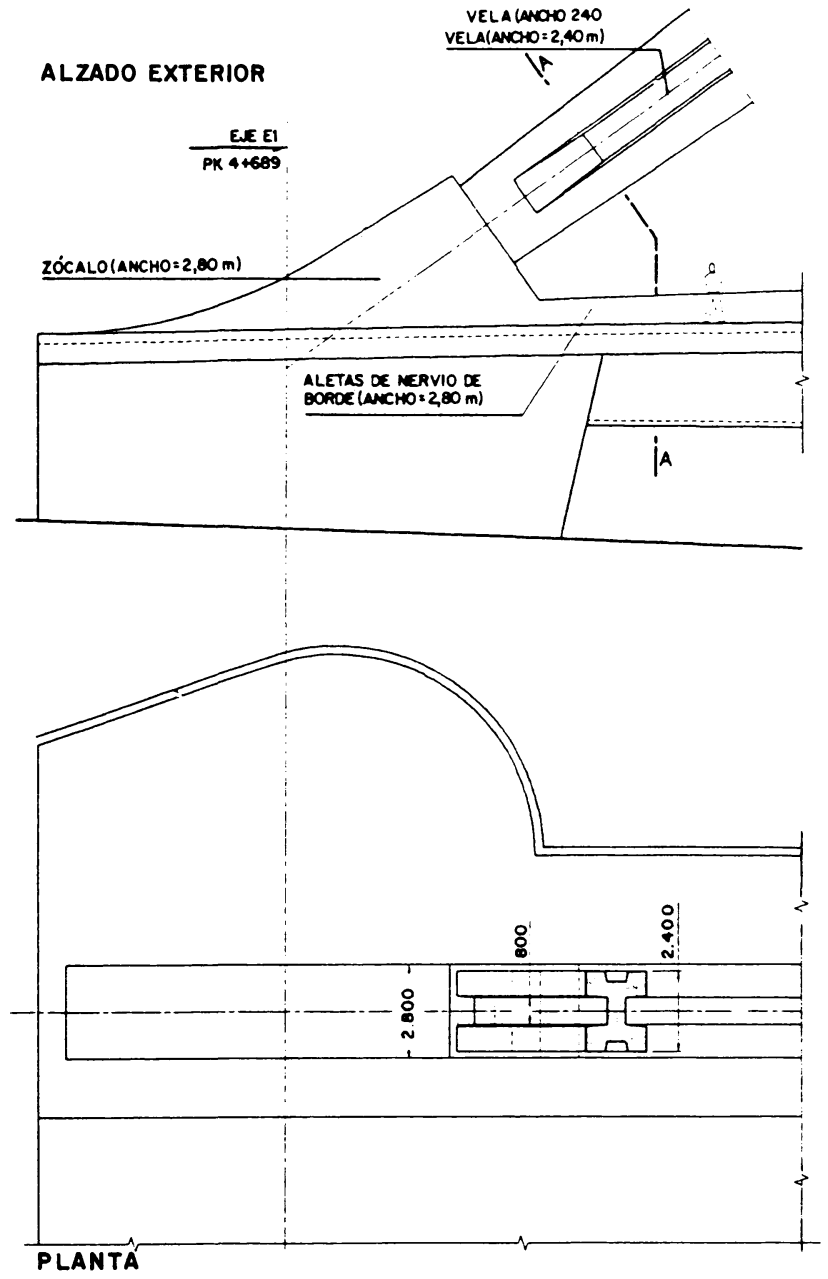

Detalle del arranque de la vela. por los largueros, las cerchas y los travesaños intermedios, enmarcado todo ello por las bandas de borde que son los nervios longitudinales.

- Planta que se enriquece con los sobreanchos de aceras que, a uno y otro lado, y con formas triangulares, resuelven la ocupación del espacio de la acera por las bases de los mástiles, los cuales, con sus puntas de flecha apuntando hacia el río, confieren a la planta del puente un sentido dinámico y que riman con las formas de la planta del contrapeso dorsal.

\section{Los apoyos del puente}

\subsection{Contrapeso dorsal}

Para suministrar la reacción descendente que requiere el extremo dorsal del puente atirantado, se planteó un importante volumen de hormigón, con planta trapecial de unos 17 metros de longitud según el eje del puente, cuyo ancho desborda ampliamente al del tablero y que se ha planteado como ensanche de la plataforma del puente, incorporando a ambos lados escaleras de descenso hasta la orilla del río. Además, esa planta trapecial incluye amplios bordes curvos que mejoran notablemente su aspecto de bloque inmenso que, gracias a un sistema de fuertes rehundidos horizontales en los alzados acaba transmitiendo una sensación de gran limpieza geométrica y de total serenidad de formas. Nada que ver, por tanto, con un simple macizo de hormigón. 
Al final, y atendiendo a una sugerencia del constructor, el contrapeso se ha modificado pasándolo de cuerpo macizo a gran caja de hormigón armado con huecos internos llenos de material granular, lo que, para mantener el margen de seguridad deseado frente al despegue, ha exigido reajustar algo sus dimensiones.

El contrapeso se cimenta en un conjunto de 15 pilotes de $180 \mathrm{~cm}$ excavados y empotrados en las areniscas del mioceno.

\subsection{Pilas}

Son cuerpos robustos en forma de prismatoide que, como plintos de escasa altura y justo debajo de cada mástil, dan apoyo al nervio de borde. Sus dimensiones, del orden de 450 por $780 \mathrm{~cm}$ en coronación, permiten alojar sendas placas de apoyo de neopreno confinado con dispositivos de deslizamiento teflón-inox, cada una de las cuales acepta una carga vertical máxima de 6.000 toneladas. El aire de esas placas no se sitúa en el plano medio del nervio de borde sino que, para tener en cuenta el par lateral que ese nervio de borde transmite a la cabeza de pila, se descentra hacia el eje del puente una distancia de $30 \mathrm{~cm}$.

Cada pila se cimenta en un conjunto de 16 pilotes de 180 $\mathrm{cm}$ de diámetro.

\section{Procedimiento constructivo}

A continuación se describe, de modo sucinto, el proceso constructivo:

Las células triangulares compuestas por cada mástil-vela y el nervio de borde se ejecutan sobre cimbra y mediante trepa, con unas etapas muy estudiadas en las que se han buscado la máxima racionalidad en el uso de los paneles de encofrado fenólico y la mayor limpieza del dibujo que esas placas de encofrado generan.

El vano de 24 metros del nervio de borde entre la pila y el anclaje del primer tirante se resuelve también sobre cimbra, aunque ello exige la realización de pilotes en el río para el apoyo frontal de la misma.

A partir de ahí, la construcción de cada nervio de borde del vano principal se va a llevar a cabo mediante avance en voladizo sobre carro. Se realizarán dovelas de 9 metros de longitud, con junta de ejecución adecuadamente dispuesta para que cada dovela aloje debidamente la placa inferior de anclaje del correspondiente tirante. El tirante se tesa antes de proceder al avance del carro a la siguiente posición.
El proceso constructivo incluye sucesivamente retesados de los dos últimos tirantes de cada fase y un retesado general del conjunto de tirantes, una vez aplicada la totalidad de cargas de peso propio.

Sigue la instalación de cerchas transversales entre nervios de borde soldándose a placas de acero que han quedado embutidas en la superficie de hormigón.

Se colocan los largueros de acero entre cerchas.

Se instalan los travesaños transversales entre cada par de cerchas.

Se montan las placas prefabricadas de hormigón y se hormigonan las bandas in situ dispuestas sobre platabandas de vigas de acero, lográndose la integridad total de la estructura.

\section{El hormigón utilizado en la obra}

Tratándose de una estructura de hormigón y bastante excepcional, la preocupación no puede limitarse a la resistencia del material sino que ha de incluir el concepto más ambicioso de durabilidad.

En cuanto a resistencia, se ha exigido en el tablero hormigón de calidad $\mathrm{H}-450$ y en el mástil, la cabeza y vela, $\mathrm{H}-600$, que, como es bien sabido, no puede hoy denominarse hormigón de alta resistencia o altas prestaciones, pero que se sale del rango usual de los hormigones estructurales que se utilizan en España. Se deseaba, además, conseguir esa resistencia sin la presencia de aditivos tipo microsílice que oscurecen el hormigón ya que, por razones estéticas, se quería un hormigón lo más blanco posible. Y, por otro lado, se trataba de conseguir un hormigón trabajable que sufriese la mínima retracción, evitándose de ese modo las fisuras de afogarado, que, precisamente en las paredes de los mástiles, conducen con facilidad a "pieles de lagarto" en el hormigón y a una segura degradación a lo largo del tiempo. Se trata de optimizar la composición del material, no sólo en lo que se refiere a resistencia a compresión, sino en todo un conjunto de direcciones que incluyen la resistencia a tracción, la retracción, la densidad del material, el riesgo de futura carbonatación y, al final, la durabilidad de la obra.

A la vista de la importancia de los valores en juego, se ha recurrido al asesoramiento del ingeniero D. Marcos Bollati, quien, en contacto directo con los constructores ( $y$, de modo específico, con Zarzuela, que es quien fabrica el hormigón), ha llevado a cabo los estudios previos de su composición. La situación se resume de modo simple en el siguiente cuadro:

\begin{tabular}{|c|c|c|c|}
\hline HORMIGÓN & $\mathrm{kg}$ CEMENTO $/ \mathrm{m}^{3}$ & $\mathrm{f}_{\mathrm{cm}} 3$ días & $\mathrm{f}_{\mathrm{cm}} 28$ días \\
\hline $\mathrm{H}-450$ & $360(405)$ & 550 & 780 \\
\hline $\mathrm{H}-600$ & 450 & 670 & 810 \\
\hline
\end{tabular}



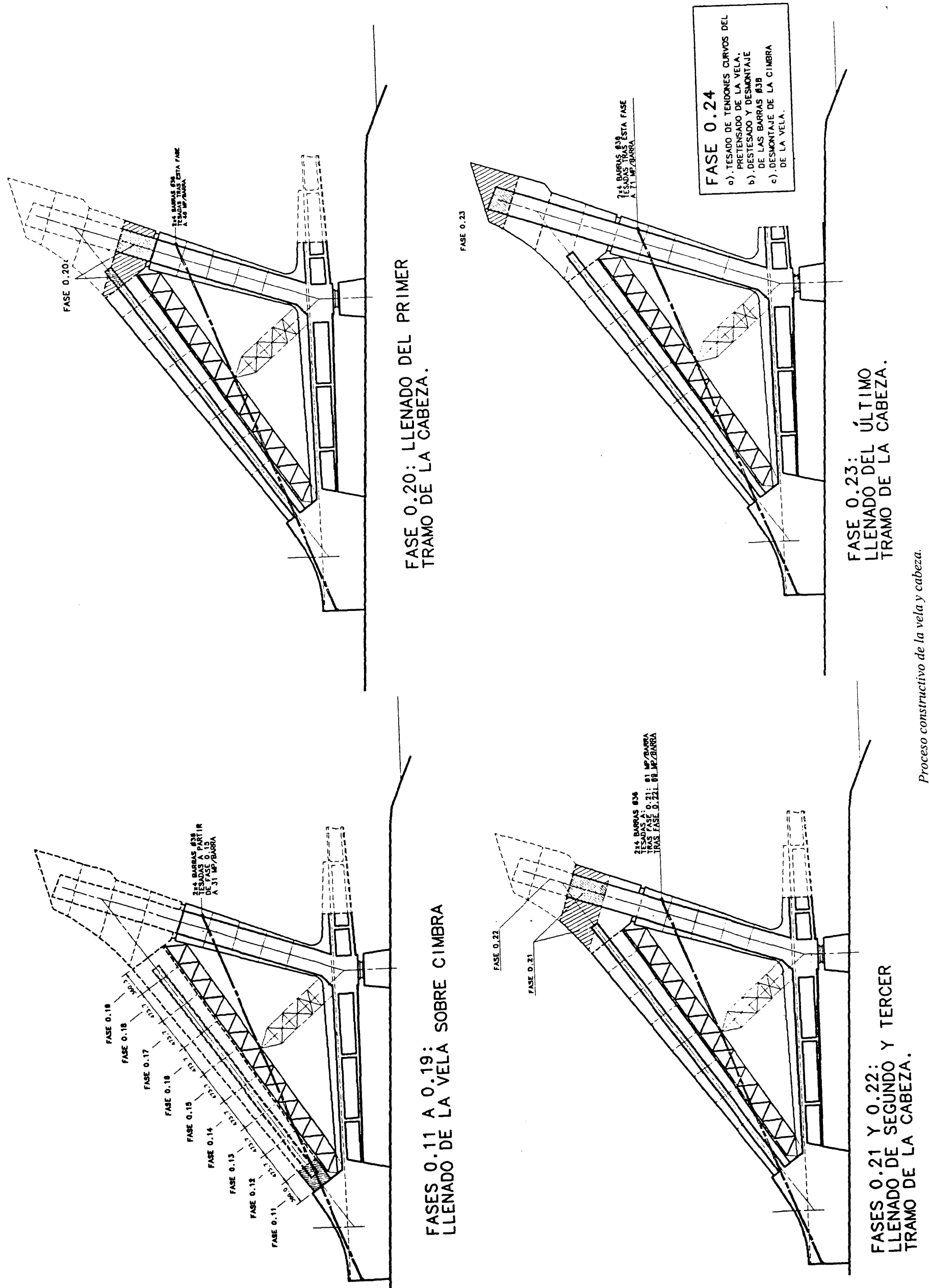


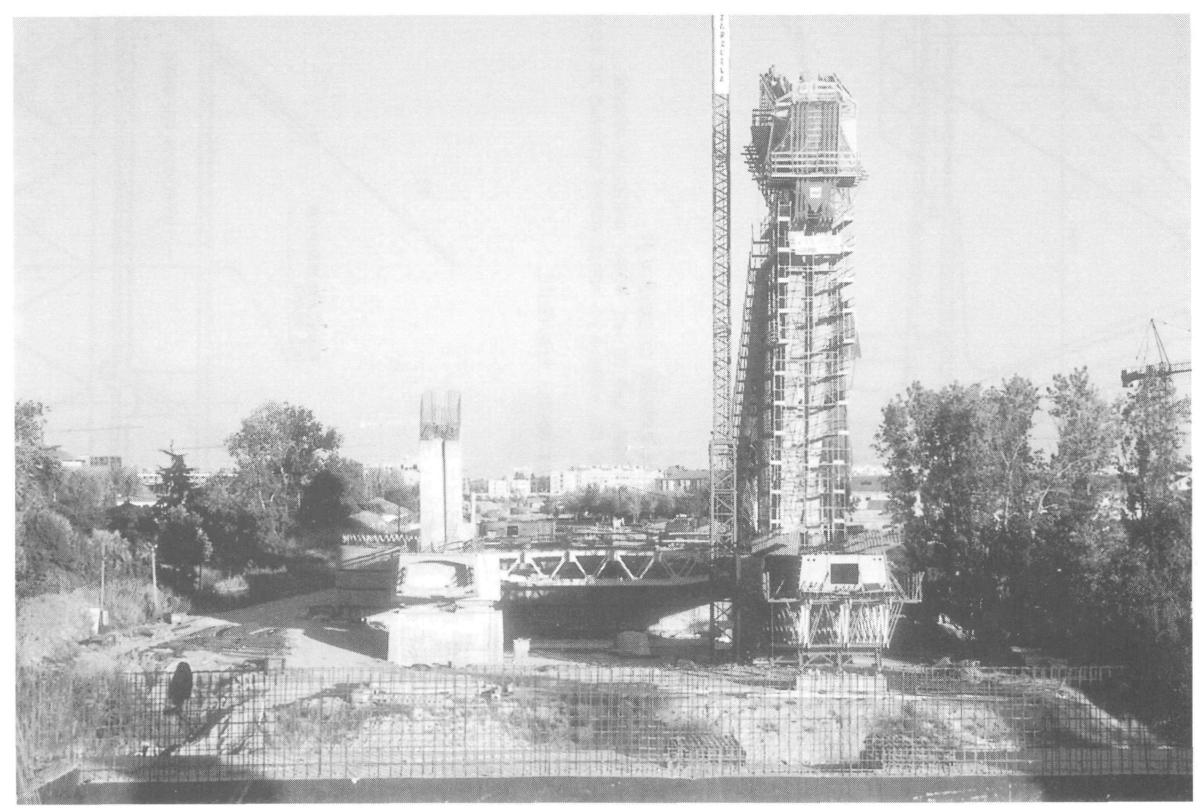

Mástil-vela.

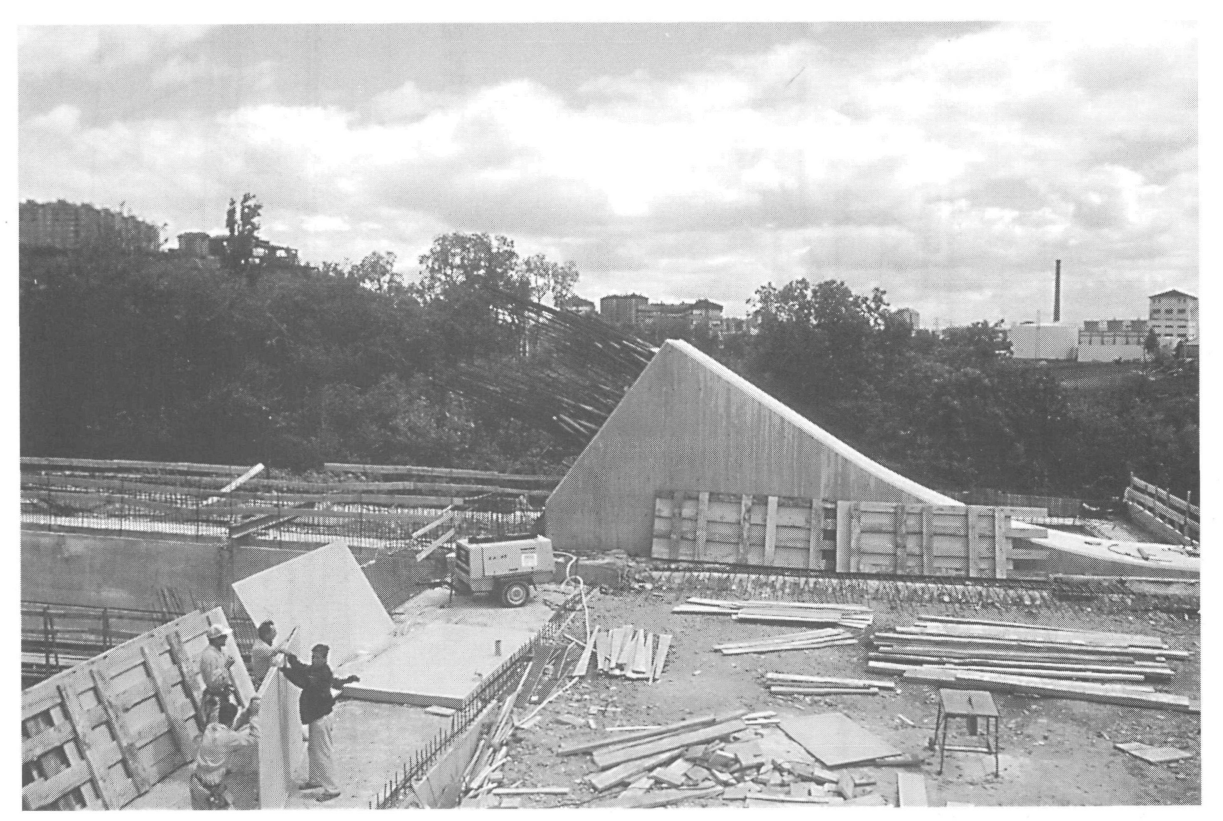

Arranque de la vela.

La dosificación de $405 \mathrm{~kg}$ de cemento por $\mathrm{m}^{3}$ ha sido al final necesaria, no por condiciones de resistencia sino de trabajabilidad del hormigón. Se constata la existente y muy alta relación entre la resistencia alcanzada y el consumo de cemento, que, desde luego, se reduce en economía, pero que conlleva todo tipo de ventajas. Entre ellas la ausencia total de fisuras en las paredes del nervio y del mástil. 


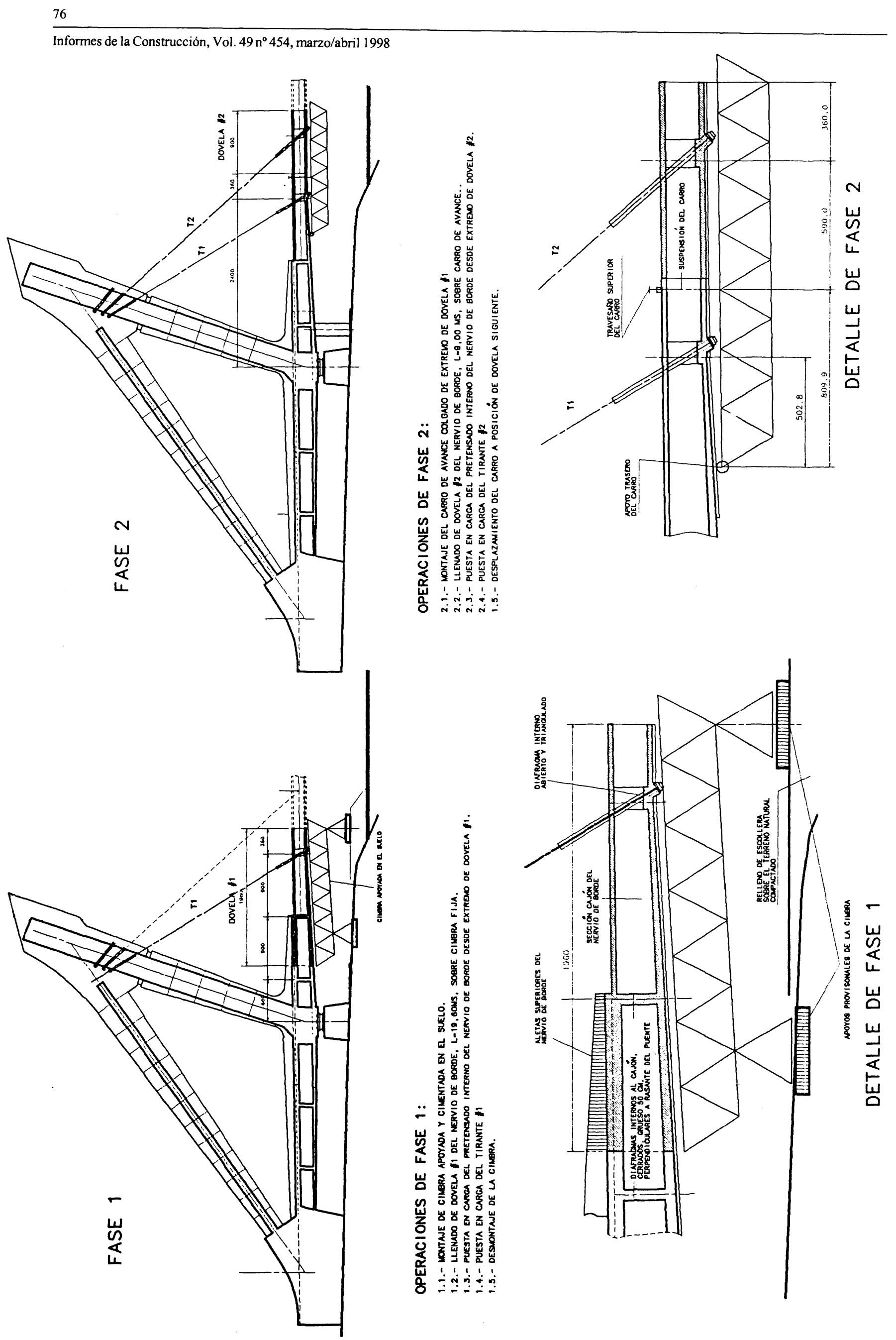

